\title{
Actitudes hacia la estadística en residentes de medicina que cursan un posgrado de investigación
}

\author{
Javier SANTABÁRBARA, Raúl LÓPEZ-ANTÓN
}

Introducción. La bioestadística es fundamental en la medicina basada en la evidencia y, por ello, para que los nuevos médicos adquieran habilidades clave en su carrera.

Objetivos. Documentar las actitudes hacia la estadística y explorar sus determinantes en los estudiantes de posgrado de medicina.

Sujetos y métodos. Estudio observacional, transversal y analítico en médicos residentes que cursaban estudios de posgrado en investigación en la Universidad de Zaragoza y respondieron a la versión en castellano del Survey of Attitudes Toward Statistics-28 (SATS-28).

Resultados. Respondió el 93,2\% $(n=41)$ de los asistentes. La puntuación media del SATS-28 fue de 4,43 $\pm 0,72$. Las puntuaciones en las subescalas indicaron que los estudiantes conciben la estadística como muy útil pero difícil. La regresión múltiple mostró que las mujeres tenían mejor actitud hacia la estadística, obteniendo puntuaciones totales 0,5 puntos más altas que los hombres $(p=0,046)$.

Conclusiones. Los residentes de medicina tienen una buena actitud hacia la estadística, aunque se necesita más investigación centrada en los cambios en la metodología de enseñanza, desde el comienzo del grado, para mejorar la percepción sobre su dificultad.

Palabras clave. Estadística. Medición de actitudes. Metodología docente. Posgrado. Residentes de medicina.

\section{Attitudes toward statistics in medicine residents studying a research postgraduate degree}

Introduction. Biostatistics is core in evidence-based medicine and thus for novel doctors to acquire key skills in their future career.

Aims. To document attitudes towards statistics and to explore its determinants in postgraduate medical students.

Subjects and methods. Observational, cross-sectional and analytical study in resident doctors who were pursuing a postgraduate degree in research at the University of Zaragoza who responded to the Spanish version of the Survey of Attitudes Toward Statistics-28 (SATS-28).

Results. The $93.2 \%(n=41)$ of attendees responded. SATS-28 average score was $4.43 \pm 0.72$. The scores on the sub-scales indicated that students conceive Statistics as very useful but difficult. Multiple regression showed that women had better attitudes towards statistics, obtaining a total score 0.5 points higher than men $(p=0.046)$.

Conclusions. Medicine residents have good attitude towards statistics, although more research focused on changes in teaching methodology is needed, even since the beginning of the degree, to improve the perception about its difficulty.

Key words. Attitudes assessment. Postgraduate. Residents of medicine. Statistics. Teaching methodology.

\section{Introducción}

La bioestadística (o estadística médica) ocupa un lugar preferente en la medicina basada en la evidencia ya que habilita al profesional médico para evaluar críticamente la evidencia médica publicada y mejora la toma de decisiones complejas en la práctica clínica diaria. Por tanto, es importante que los médicos jóvenes adquieran las competencias nece- sarias en bioestadística para su futuro desempeño profesional.

La actitud de los estudiantes hacia la bioestadística contribuye al éxito en esta materia, un tema bien estudiado en el Grado de Medicina [1]. Se ha publicado que los estudiantes de medicina presentan una ansiedad moderada durante el estudio de la bioestadística [2], lo que podría dificultar su aprendizaje [3] y su generalización a la práctica médica
Departamento de Microbiología, Medicina Preventiva y Salud Pública (J. Santabárbara). Departamento de Psicología y Sociología (R. LópezAntón). Universidad de Zaragoza. Zaragoza, España.

Correspondencia:

Dr. Javier Santabárbara Serrano. Departamento de Microbiología, Medicina Preventiva y Salud Pública. Facultad de Medicina. Universidad de Zaragoza. Domingo Miral, s/n. E-50009 Zaragoza.

E-mail:

jsantabarbara@unizar.es

Recibido:

30.11.18.

Aceptado:

12.12.18.

Conflicto de intereses:

No declarado.

Competing interests:

None declared.

(C) 2019 FEM

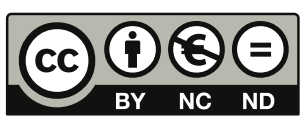

Artículo open access bajo la licencia CC BY-NC-ND (https:// creativecommons.org/licenses/ by-nc-nd/4.0/).

ISSN: 2014-9832

ISSN (ed. digital): $2014-9840$ 
Tabla I. Cuestionario sobre las actitudes hacia la estadística (SATS-28).

1. Me gusta la estadística

2. Me siento inseguro cuando hago problemas de estadística

3. No entiendo mucho la estadística debido a mi manera de pensar

4. Las fórmulas estadísticas son fáciles de entender

5. La estadística no sirve para nada

6. La estadística es una asignatura complicada

7. La estadística es un requisito en mi formación como profesional

8. Mis habilidades estadísticas me facilitarán el acceso al mundo laboral

9. No tengo ni idea de qué va la estadística

10. La estadística no es útil para el profesional común

11. Me siento frustrado al hacer pruebas de estadística

12. Los conceptos estadísticos no se aplican fuera del trabajo

13. Utilizo la estadística en la vida cotidiana

14. En las clases de estadística estoy en tensión

15. Disfruto en clase de estadística

16. Las conclusiones estadísticas raramente se dan en la vida

17. La mayoría de la gente aprende estadística rápidamente

18. Aprender estadística requiere mucha disciplina

19. En mi profesión no usaré estadística

20. Cometo muchos errores matemáticos cuando hago estadística

21. Me da miedo la estadística

22. La estadística implica mucho cálculo

23. Puedo aprender estadística

24. Entiendo las formulas estadísticas

25. La estadística no es importante en mi vida

26. La estadística es muy técnica

27. Me resulta difícil comprender los conceptos estadísticos

28. La mayoría de la gente debe cambiar su manera de pensar para hacer estadística

Grado de acuerdo con cada una de las aseveraciones: de 1 ('totalmente en desacuerdo') a 7 ('totalmente de acuerdo'). una vez egresados, ya que no poseen los conocimientos necesarios [4]. Una manera de suplir la carencia de conocimientos en esta materia es cursando estudios de posgrado.

A pesar de su importancia, son escasos los trabajos realizados en estudiantes de posgrado [5] o residentes de hospital [6] en la literatura médica, siendo un problema aún no abordado en nuestro país.

En este contexto, nos planteamos los siguientes objetivos: documentar las actitudes hacia la estadística de estudiantes de posgrado y explorar los determinantes de las actitudes hacia esta materia.

\section{Sujetos y métodos}

\section{Diseño, población en estudio y selección de la muestra}

Estudio observacional, de corte transversal y analítico. Los participantes en el estudio fueron médicos residentes que cursaban un máster de iniciación a la investigación en la Facultad de Medicina de la Universidad de Zaragoza. La selección se realizó mediante un muestreo no probabilístico de conveniencia. Los datos se recogieron al comienzo de dicho máster, en noviembre de 2018.

\section{Instrumentos}

Las características sociodemográficas (edad y sexo), profesionales (especialidad médica y año de residencia) y conocimientos previos de bioestadística (realización de cursos y manejo de programa estadístico) se recogieron mediante un cuestionario realizado ad hoc para este estudio.

Para recoger las actitudes hacia la estadística, se usó la versión en castellano del SATS-28 (Survey of Attitudes Toward Statistics-28) [7] (Tabla I), cuestionario que evalúa las actitudes hacia la estadística en cuatro subescalas: afecto (actitudes positivas y negativas hacia la estadística), competencia cognitiva (actitudes sobre el conocimiento y habilidades en estadística), valor (actitudes sobre cuánto valor tienen las estadísticas en la vida diaria y profesional) y dificultad (actitudes sobre la dificultad de la estadística). El evaluado debe calificar cada ítem en una escala tipo Likert de 1 ('muy en desacuerdo') a 7 ('muy de acuerdo'). Cuanto mayor sea la puntuación en SATS-28 o cualquier subescala, más positiva será la actitud hacia la estadística.

\section{Análisis estadístico}

La normalidad de las variables en estudio se evaluó 
mediante el contraste de Shapiro-Wilk para muestras pequeñas. La distribución de la puntuación (total y subescalas) en el SATS-28 se ajustó a una normal.

Se empleó la prueba $t$ de Student para una muestra con el objetivo de contrastar si la puntación media en el SATS-28 y cada una de sus subescalas difiere de 4 (puntuación neutral en una escala de 7).

La comparación de las puntuaciones en las actitudes hacia la estadística según las características sociodemográficas, profesionales y conocimientos previos de bioestadística de la muestra de estudio se presentó mediante la media \pm desviación estándar, utilizando el contraste paramétrico $t$ de Student para la comparación de dos muestras independientes.

Finalmente, para estimar el efecto de las características sociodemográficas, profesionales y conocimientos previos de bioestadística en las puntuaciones en la escala de las actitudes hacia la estadística, se utilizó el modelo de regresión lineal multivariado. El porcentaje de la variabilidad en la puntuación total de la escala de las actitudes hacia la estadística (SATS-28) según las características sociodemográficas, profesionales y conocimientos previos de bioestadística se calculó utilizando el coeficiente de determinación lineal $R^{2}$. Se comprobaron las condiciones de aplicación de regresión lineal.

El análisis de los datos se llevó a cabo mediante el programa estadístico $R$ v. 3.5.1.

\section{Aspectos éticos}

El decano de la Facultad de Medicina de la Universidad de Zaragoza aprobó el protocolo del estudio y todos los participantes firmaron un consentimiento informado. Los cuestionarios se rellenaron de forma anónima.

\section{Resultados}

La encuesta fue cumplimentada por 41 de los 44 asistentes (93,2\%). La edad media de los residentes fue de $26 \pm$ 7,7 años, siendo el 70,7\% mujeres. En relación a sus características profesionales, el $78 \%$ pertenecían a una especialidad clínica y la mayoría eran residentes de primer año (63,4\%). El 25\% de ellos, aproximadamente, habían realizado un curso previo de bioestadística, pero apenas un 5\% manejaba algún programa estadístico.

En cuanto a las puntuaciones de las subescalas del SATS-28, así como la puntuación global del cuestionario, la mayoría de los posgraduados médicos mantuvieron actitudes positivas hacia la esta-
Tabla II. Comparación de la puntuación total en SATS-28 según características sociodemográficas, profesionales y relativas a conocimientos previos en bioestadística.

\begin{tabular}{|c|c|c|c|c|}
\hline & & & Media $\pm \mathrm{DE}$ & $p^{a}$ \\
\hline \multirow{4}{*}{$\begin{array}{l}\text { Características } \\
\text { sociodemográficas }\end{array}$} & \multirow[b]{2}{*}{ Edad } & $<25$ años $(n=22)$ & $4,55 \pm 0,72$ & \multirow[b]{2}{*}{0,253} \\
\hline & & $\geq 25$ años $(n=19)$ & $4,29 \pm 0,71$ & \\
\hline & \multirow[b]{2}{*}{ Sexo } & Varón ( $n=12)$ & $4,20 \pm 0,62$ & \multirow[b]{2}{*}{0,194} \\
\hline & & Mujer $(n=29)$ & $4,52 \pm 0,75$ & \\
\hline \multirow{4}{*}{$\begin{array}{l}\text { Características } \\
\text { profesionales }\end{array}$} & \multirow{2}{*}{ Especialidad } & Quirúrgica $(n=9)$ & $4,46 \pm 0,92$ & \multirow{2}{*}{0,879} \\
\hline & & Clínica $(n=32)$ & $4,42 \pm 0,67$ & \\
\hline & \multirow[b]{2}{*}{ Años de residencia } & 1 año $(n=26)$ & $4,60 \pm 0,79$ & \multirow[b]{2}{*}{0,038} \\
\hline & & $\geq 2$ años $(n=15)$ & $4,12 \pm 0,48$ & \\
\hline \multirow{4}{*}{$\begin{array}{l}\text { Conocimientos } \\
\text { previos de } \\
\text { bioestadística }\end{array}$} & \multirow{2}{*}{ Realización de cursos } & No $(n=31)$ & $4,53 \pm 0,72$ & \multirow{2}{*}{0,093} \\
\hline & & Sí $(n=10)$ & $4,09 \pm 0,66$ & \\
\hline & \multirow{2}{*}{$\begin{array}{l}\text { Manejo de } \\
\text { programa estadístico }\end{array}$} & No $(n=39)$ & $4,38 \pm 0,70$ & \multirow{2}{*}{0,065} \\
\hline & & Sí $(n=2)$ & $5,34 \pm 0,69$ & \\
\hline
\end{tabular}

DE: desviación estándar. ${ }^{a} t$ de Student.

dística, de modo que la media del SATS-28 fue de $4,43 \pm 0,72$ puntos, siendo significativamente superior al neutral $(p<0,001)$. Además, las puntuaciones en la subescala de competencia cognitiva (media: 4,86 $\pm 0,93 ; p<0,001$ ) indicaron que los estudiantes tenían conocimientos y habilidades básicas cuando aprendían y aplicaban la estadística. La puntuación alta en la subescala de valor (media: 5,53 \pm 0,$76 ; p<0,001$ ) indicó que los estudiantes conciben la estadística como muy útil en su vida personal y profesional. Por otra parte, la puntuación baja en la subescala de afecto (media: 3,98 $\pm 1,29$ ) indicó una tendencia a la ausencia de sentimientos positivos hacia la estadística $(p=0,936)$. Del mismo modo, la subescala de dificultad tuvo una puntuación baja (media: 3,33 $\pm 0,72$ ), significativamente inferior a la neutral $(p<0,001)$, es decir, los estudiantes pensaban que la estadística es una materia difícil.

La tabla II muestra la comparación de las puntuaciones totales en el SATS-28 según las variables en estudio. No se encontraron diferencias estadísticamente significativas en las actitudes hacia la estadística según características sociodemográficas (edad y sexo). En relación a las características profesionales, aquellos que cursaron su primer año de residen- 
Tabla III. Regresión lineal múltiple de la puntuación total del SATS-28 según variables sociodemográficas, profesionales y conocimientos previos de bioestadística.

\begin{tabular}{|c|c|c|}
\hline & $b$ (IC 95\%) & $p$ \\
\hline $\operatorname{Edad}\left(\geq 25\right.$ años) ${ }^{a}$ & $-0,31(-0,89$ a 0,28$)$ & 0,292 \\
\hline Sexo (mujer) & $0,51(0,01$ a 1,02) & 0,046 \\
\hline Especialidad (clínica) ${ }^{\mathrm{b}}$ & $0,17(-0,36$ a 0,69$)$ & 0,523 \\
\hline Años de residencia (> 1 año) c & $-0,68(-1,29 a-0,07)$ & 0,030 \\
\hline Curso previo de bioestadística (sí) & $-0,16(-0,70$ a 0,37$)$ & 0,542 \\
\hline Manejo de programa estadístico (sí) & $1,17(0,16$ a 2,18$)$ & 0,024 \\
\hline$R^{2}$ & 0,303 & \\
\hline
\end{tabular}

Los residuos del modelo siguieron distribución normal (valor $p$ en el contraste de Shapiro-Wilk =0,489). b: coeficiente de regresión; IC 95\%: intervalo de confianza al 95\%; $R^{2}$ : coeficiente de determinación del modelo. a Referencia: < 25 años. ${ }^{b}$ Referencia: quirúrgica. ' Referencia: primer año de residencia.

cia $(p=0,038)$ y que manejaban un programa estadístico ( $p=0,065$, marginalmente significativo) manifestaron actitudes más positivas hacia la estadística. Por el contrario, no hallamos diferencias significativas en la puntuación total del SATS-28 según la especialidad y la realización de cursos previos de bioestadística.

La tabla III muestra los resultados de la regresión múltiple de los predictores de la puntuación total de la actitud hacia la estadística. Los resultados mostraron que las mujeres tuvieron una puntuación total 0,5 puntos superior a la de los varones $(p=0,046)$. La edad no alcanzó la significación estadística, pero mostró una tendencia negativa (a mayor edad, menor actitud positiva hacia la estadística). Aquellos residentes de segundo año o superior tuvieron una puntuación total 0,68 puntos inferior a la de los residentes de primer año $(p=0,030)$. Esto es, los residentes de primer año mostraron una mayor actitud positiva que los residentes mayores. No se encontró significación estadística respecto a la especialidad médica. En relación a los conocimientos previos de bioestadística, aquellos con conocimientos sobre manejo de un programa estadístico obtuvieron 1,2 puntos más en el SATS-28 respecto a aquellos sin dichos conocimientos $(p=0,024)$, lo que se traduce en una actitud más positiva hacia la estadística. Sin embargo, la realización de un curso previo en bioestadística no tuvo efecto sobre la puntuación total en el SATS-28.

\section{Discusión}

Los resultados del primer estudio en alumnos de posgrado universitario en una facultad de medicina española sugieren que los residentes poseen una actitud positiva hacia la estadística, en términos generales, aunque la perciben como una materia difícil.

Basándonos en los resultados del modelo de regresión y en relación al sexo de los participantes, no encontramos diferencias significativas en las actitudes totales hacia la estadística. En relación a la edad, en nuestro estudio, los residentes de mayor edad muestran una tendencia hacia actitudes más negativas que los jóvenes; estos resultados son similares a la bibliografía previa [5,6]. Respecto a la experiencia en la formación de especialización, no hallamos diferencias significativas en las actitudes hacia la estadística de los residentes de primer año respecto a los residentes con más experiencia, aunque sí es reseñable que estos últimos muestran una actitud menos positiva hacia la estadística. En relación a las especialidades, los estudiantes de especialidades clínicas mostraron mejores actitudes que los de quirúrgicas, aunque sin alcanzar la significación estadística, quizá porque entre los primeros es más frecuente el uso de herramientas estadísticas. Estos resultados coinciden con el estudio de Harshe et al [6], aunque son contrarios a un estudio chino [5], que sí obtiene diferencias en relación a la especialidad.

Respecto al conocimiento previo de un programa estadístico, Zhang et al [5] defienden que, dado que la estadística implica cálculos complejos, los estudiantes con una mejor base informática deberían tener más confianza en los aspectos computacionales de la estadística; por nuestra parte, hemos encontrado una asociación positiva entre el manejo de un programa estadístico y mejores actitudes hacia la estadística. Sin embargo, y de forma contradictoria al estudio chino, resulta sorprendente que aquellos que realizaron cursos previos en bioestadística mostraron una tendencia más negativa hacia la estadística. Algunos autores [8] sugieren que es improbable que la realización previa de cursos independientes en bioestadística rectifique las actitudes de los profesionales clínicos hacia la investigación.

Nuestros hallazgos sugieren que los residentes en medicina conciben la estadística como una materia difícil. La solución a este problema podría pasar por una docencia que evite el uso de terminología estadística y complejas fórmulas matemáticas, con un mayor uso de nuevas tecnologías y mayor importancia del aprendizaje basado en problemas y la utilización de casos prácticos [9]. 
No obstante, hemos de señalar algunas debilidades: la principal limitación es la utilización de muestreo no probabilístico de conveniencia en un único centro y con escaso tamaño muestral, lo que imposibilita la generalización de los hallazgos. Otra limitación es que el SATS-28 no está validado estrictamente en población española, aunque sí internacionalmente para estudiantes de posgrado [10].

En conclusión, los residentes en medicina poseen una buena actitud hacia la estadística, aunque la perciben como una materia difícil. Por tanto, ya desde el grado habría que evitar la percepción de la estadística como una materia compleja mediante el uso sistemático de ejemplos reales, aprendizaje basado en problemas, etc. Se necesitan futuros estudios que se encaminen a documentar una mejora en la actitud hacia la estadística mediante la implementación de dichas técnicas desde el inicio de los estudios de grado.

\section{Bibliografía}

1. Milic NM, Masic S, Milin-Lazovic J, Trajkovic G, Bukumiric Z Savic M, et al. The importance of medical students' attitudes regarding cognitive competence for teaching applied statistics: multi-site study and meta-analysis. PLoS One 2016; 11: e0164439.

2. Beurze SM, Donders ART, Zielhuis GA, De Vegt F, Verbeek ALM. Statistics anxiety: a barrier for education in research methodology for medical students? Med Sci Educ 2013; 23: 377.

3. Windish DM, Huot SJ, Green ML. Medicine residents' understanding of the biostatistics and results in the medical literature. JAMA 2007; 298: 1010-22.

4. Torales J, Barrios B, Viveros-Filártiga D, Giménez-Legal E, Samudio M, Aquino S, et al. Conocimiento sobre métodos básicos de estadística, epidemiología e investigación de médicos residentes de la Universidad Nacional de Asunción, Paraguay. Educ Med 2017; 18: 226-32.

5. Zhang Y, Shang L, Wang R, Zhao Q, Li C, Xu Y, et al. Attitudes toward statistics in medical postgraduates: measuring, evaluating and monitoring. BMC Med Educ 2012; 23: 117.

6. Harshe DG, Abraham DA. A study of attitudes of teaching faculty and postgraduate residents at a tertiary care teaching hospital toward biostatistics. Muller Journal of Medical Sciences and Research 2017; 8:10-4.

7. Figueroa SM, Pérez MA, Bacelli S, Prieto G, Moler E. Actitudes hacia la estadística en estudiantes de ingeniería. Interdisciplinaria 2012; 29: 37-49.

8. West CP, Ficalora RD. Clinician attitudes toward biostatistics. Mayo Clinic Proc 2007; 82: 939-43.

9. Miles S, Price GM, Swift L, Shepstone L, Leinster SJ. Statistics teaching in medical school: opinions of practising doctors. BMC Med Educ 2010; 10: 75.

10. Maat SM, Rosli MK. Validating the attitude towards statistics instrument for postgraduate students. Advanced Science Letters 2018; 24: 3432-5. 